\title{
Color Superfluidity and "Baryon" Formation in Ultracold Fermions
}

\author{
Ákos Rapp ${ }^{1,2}$, Gergely Zaránd ${ }^{1,2}$, Carsten Honerkamp ${ }^{3}$, and Walter Hofstetter ${ }^{4}$ \\ 1 Theoretical Physics Department, Institute of Physics, \\ Budapest University of Technology and Economy, Budapest, H-1521, Hungary, \\ 2 Institut für Theoretische Festkörperphysik,Universität Karlsruhe, D-76128 Karlsruhe, Germany, \\ ${ }^{3}$ Theoretical Physics, Universität Würzburg, D-97074 Würzburg, Germany \\ 4 Institut für Theoretische Physik, Johann Wolfgang Goethe-Universität, 60438 Frankfurt/Main, Germany
}

(Dated: February 6, 2008)

\begin{abstract}
We study fermionic atoms of three different internal quantum states (colors) in an optical lattice, which are interacting through attractive on site interactions, $U<0$. Using a variational calculation for equal color densities and small couplings, $|U|<\left|U_{C}\right|$, a color superfluid state emerges with a tendency to domain formation. For $|U|>\left|U_{C}\right|$, triplets of atoms with different colors form singlet fermions (trions). These phases are the analogies of the color superconducting and baryonic phases in QCD. In ultracold fermions, this transition is found to be of second order. Our results demonstrate that quantum simulations with ultracold gases may shed light on outstanding problems in quantum field theory.
\end{abstract}

PACS numbers: 03.75.Mn, 32.80.Pj, 71.35.Lk

The achievement of Bose-Einstein condensation (BEC) a decade ago has opened the new field of ultracold atomic physics where dilute alkali-metal gases are cooled into the quantum degenerate regime [1]. In particular, degenerate Fermi gases have been realized [2] with temperatures down to $T / T_{F} \approx 0.05$. An attractive feature of these systems is the high degree of tunability. Feshbach resonances allow atomic interaction strengths to be tuned [3], while optical lattices can be used to create artificial crystals of light in which atoms form Bloch bands like electrons in solids [4, 5, 6]. Moreover, the effective interaction strength can be modulated by the optical lattice alone and thus tuned through quantum phase transitions like the bosonic Mott transition [6]. Recently, fermionic ${ }^{40} \mathrm{~K}$ atoms have been loaded into optical lattices as well 7]. It has been predicted that quantum simulations with cold fermions in optical lattices may ultimately shed light on complex solid-state phenomena like high-temperature superconductivity [8]. In a different line of experiments, fermionic superfluids close to the BEC-BCS transition have been created and even loaded into optical lattices [9, 10]. Very recently, also pairing of fermions with unequal spin densities and the eventual breakdown of superfluidity has been studied [11].

More generally, with the degrees of freedom offered by cold atoms, it is possible to create new states of matter which have no equivalent in condensed matter. While typical electronic systems have at most $\mathrm{SU}(2)$ spin rotational symmetry, the atomic total angular momentum $F$ can be larger than $1 / 2$, resulting in $2 F+1$ hyperfine states. In optical traps, all of these states can be trapped, e.g., for spinor condensates of ${ }^{23} \mathrm{Na}$ and ${ }^{87} \mathrm{Rb}$ [12]. For fermionic atoms, coexistence of 3 different hyperfine states of ${ }^{40} \mathrm{~K}$ in an optical trap has been demonstrated [13].
Here, we study optical lattices loaded with fermionic atoms that possess three different internal quantum numbers (colors), $\alpha=1 \ldots 3$ and interact through an attractive on-site interaction. This system is well described by a SU(3) Hubbard Hamiltonian. For small interactions a color superfluid (CSF) state appears with a triplet order parameter [14]. Here we show that for low fillings and larger interactions a quantum phase transition takes place from the superfluid state to a Fermi liquid phase, where groups of three fermions bind together to form trions. This transition is closely analogous to the one conjectured in QCD, where at large quark densities, a color superconducting state is expected while at low densities, baryonic matter emerges.

Such an optical system can be realized, e.g., by loading ${ }^{6} \mathrm{Li}$ atoms of nuclear spin $I=1$ into an optical lattice and applying a magnetic field that is larger than the hyperfine coupling. In this case, the electron spin of the Li atom is essentially polarized along the external field direction and the $2 I+1$ nuclear quantum numbers provide the internal degrees of freedom. Attractive interactions independent of the nuclear spin are induced by an anomalously large and negative triplet scattering length $a_{s}=-2160 a_{0}$ [15]. We expect the experimentally accessible temperatures for this three-state ensemble to be in the same range as for two-component mixtures, i.e., $T / T_{F} \approx 0.05$ [9]. In the absence of spin-flip processes, the densities of the three "color" states can be adjusted independently by radiofrequency sweeps and selective evaporation [11].

In an optical lattice the atoms experience a periodic potential $V(x)=V_{0} \sum_{l=x, y, z} \cos ^{2}\left(k x_{l}\right)$, with $k$ the wave vector of the laser and the directions labeled by $l$. At low fillings, this system can be described with good accuracy 
by the Hubbard Hamiltonian

$$
H=-t \sum_{i, j, \alpha} \hat{c}_{i \alpha}^{\dagger} \hat{c}_{j \alpha}+\frac{U}{2} \sum_{i, \alpha \neq \beta} \hat{n}_{i \alpha} \hat{n}_{i \beta} .
$$

Here $\hat{n}_{i \alpha}=\hat{c}_{i \alpha}^{\dagger} \hat{c}_{i \alpha}$ measures the number of fermions with color $\alpha$ at site $i$, with $\hat{c}_{i \alpha}^{\dagger}$ the creation operator of a fermion. The interaction $U$ is assumed to be negative throughout this Letter while the ratio $|U| / t$ can be tuned in a wide range by changing the depth $V_{0}$ of the optical lattice. In Eq. (10), we also assume a homogeneous optical lattice, and neglect to a first approximation the parabolic confinement potential of the atom trap. Note that Eq. (1) has an SU(3) symmetry associated with global rotations, $c_{i \alpha} \rightarrow \sum_{\beta} U_{\alpha \beta} c_{i \beta}$, and $H$ conserves the total number of particles of each color, $\hat{N}_{\alpha} \equiv \sum_{i} \hat{n}_{i \alpha}$. For ${ }^{6} \mathrm{Li}$ in a strong magnetic field, this symmetry arises because the interaction between the atoms is mediated by electronic van der Waals forces, which are independent of the nuclear degrees of freedom.

For small values of $|U| / t$, the attractive interaction has been shown to induce a superfluid state at $T=0$ [14]. In this state, fermions are paired to form Cooper pairs with an order parameter $\Delta_{\alpha \beta} \equiv\left\langle c_{i \alpha} c_{i \beta}\right\rangle$ which transforms according to the adjoint representation $\overline{3}$ of $\mathrm{SU}(3)$. For very large values of $|U| / t$, however, perturbation theory in the hopping $t$ predicts a state of entirely different nature: Here three atoms tend to form a composite object, a local bound color singlet of energy $3 U$, which can hop between neighboring sites with an amplitude $t_{\text {eff }} \sim t^{3} / U^{2}$ and interact repulsively to form a Fermi liquid at very low temperatures. Note that the symmetry of the superfluid and the trionic state is different; therefore, there must be a phase transition that separates them. As we shall see, this transition is of second order, and the two states are separated by a quantum critical point.

The above transition occurs at intermediate values of $U / t$, where neither perturbation theory nor diagrammatic approaches can be applied successfully. We therefore use a variational approach in the high-dimensional limit $d \rightarrow \infty$. As a variational ansatz at $T=0$, we use a Gutzwiller-projected wave function $|G\rangle=\prod_{i} g^{\hat{t}_{i}}|B C S\rangle$, where $|B C S\rangle$ is a BCS superfluid state with two out of three color states paired

$$
|B C S\rangle=\prod_{\epsilon_{\mathbf{k}^{\prime}}<\mu_{3}} \hat{c}_{\mathbf{k}^{\prime} 3}^{+} \prod_{\mathbf{k}}\left(u_{\mathbf{k}}+v_{\mathbf{k}} \hat{c}_{\mathbf{k} 1}^{+} \hat{c}_{-\mathbf{k} 2}^{+}\right)|0\rangle,
$$

and $\hat{P}_{G} \equiv \prod_{i} g^{\hat{t}_{i}}=\prod_{i}\left[1+(g-1) \hat{n}_{i 1} \hat{n}_{i 2} \hat{n}_{i 3}\right]$ is a generalized Gutzwiller projector that favors triply occupied states for large values of the variational parameter $g$. Without making a restriction, we have assumed in Eq. (2) that only the $\Delta_{12}=-\Delta_{21}$ components of the order parameter are non-zero.This corresponds to a particular gauge of the pairing field [14]. The factors $u_{\mathbf{k}}$ and $v_{\mathbf{k}}$ denote the usual BCS coherence factors, $u_{\mathbf{k}}^{2}=\frac{1}{2}\left(1+\xi_{\mathbf{k}} / \sqrt{\xi_{\mathbf{k}}^{2}+\Delta^{2}}\right)$ and $v_{\mathbf{k}}=\sqrt{1-u_{\mathbf{k}}^{2}}$, with $\xi_{\mathbf{k}}=\epsilon_{\mathbf{k}}-\mu_{12}$, and $\epsilon_{\mathbf{k}}=-2 t \sum_{l=1}^{d} \cos k_{l}$ the kinetic energy of the atoms on the lattice.

For $n_{1}=n_{2}=n_{3}$, one can gain energy in the superfluid state by transferring particles to the channels where the superfluid condensate forms. Therefore, for a given total density $n$, the CSF has a global energy minimum for slightly unequal densities, $n_{1}=n_{2} \neq n_{3}$. In Eq. (2), we therefore introduced different chemical potentials for the first two and the third channel, $\mu_{12} \neq \mu_{3}$. Note, however, that $\mu_{12} \neq \mu_{3}$ are not our variational parameters; they are just used to fix the total density $n \equiv \sum_{\alpha=1}^{3}\left\langle\hat{n}_{i \alpha}\right\rangle$ and $n_{3} \equiv\left\langle\hat{n}_{i 3}\right\rangle$.

To obtain our variational estimate for the energy, we need to evaluate the expectation value of the Hamiltonian, $E\left(\Delta, g, n, n_{3}\right) \equiv\langle G|H| G\rangle /\langle G \mid G\rangle$, and minimize it with respect to the independent parameters $\Delta, g$ and $n_{3}$ for a given density $n$. To compute quantities of the form $\langle\hat{O}\rangle_{G} \equiv\left\langle B C S\left|\hat{P}_{G} \hat{O} \hat{P}_{G}\right| B C S\right\rangle$, we first expand the product $\hat{P}_{G}=\prod_{i}\left[1+(g-1) \hat{t}_{i}\right]$ and rewrite $\hat{P}_{G} \hat{O} \hat{P}_{G}$ so that no operator $\hat{c}_{i \alpha}^{\dagger}$ occurs to the right of the corresponding annihilation operator, $\hat{c}_{i \alpha}$. We can then use Wick's theorem to evaluate the expectation value and recast it in terms of a Grassmannian effective field theory with action:

$$
\mathcal{S}=-\frac{1}{2} \sum_{i j} \bar{\Psi}_{i}\left(D_{0}^{-1}\right)_{i j} \Psi_{j}-u \sum_{i} t_{i}
$$

Here $t_{i}=\prod_{\alpha} \bar{c}_{i \alpha} c_{i \alpha}$, with $c_{i \alpha}$ a Grassman field, and $\bar{\Psi}_{i}=\left(\bar{c}_{i \alpha}, c_{i \beta}\right)$ denotes a "Nambu spinor". The expectation value of the particle density is given by $\left\langle\hat{c}_{i \alpha}^{+} \hat{c}_{i \alpha}\right\rangle_{G}=\left\langle n_{i \alpha}\right\rangle_{\mathcal{S}}-u\left\langle t_{i}\right\rangle_{\mathcal{S}}$, while the average of terms occurring in the kinetic and interaction energy are given by $\sum_{\alpha}\left\langle\hat{c}_{i \alpha}^{+} \hat{c}_{j \alpha}\right\rangle_{G}=\sum_{\alpha}\left\langle\bar{c}_{i \alpha} c_{j \alpha}\left[1-(1-g)\left(d_{i \alpha}+d_{j \alpha}\right)+\right.\right.$ $\left.\left.(1-g)^{2}\left(d_{i \alpha} d_{j \alpha}+\delta_{i j} d_{i \alpha}\right)\right]\right\rangle_{\mathcal{S}}$, and $\left\langle\hat{n}_{i \alpha} \hat{n}_{i \beta}\right\rangle_{G}=\left\langle n_{i \alpha} n_{i \beta}\right\rangle_{\mathcal{S}}-$ $u\left\langle t_{i}\right\rangle_{\mathcal{S}}$, with $n_{i \alpha}=\bar{c}_{i \alpha} c_{i \alpha}$ and $d_{i \alpha}=\prod_{\beta \neq \alpha} n_{i \beta}$. The Gutzwiller projector manifests in Eq. (3) as an effective interaction, $u=1-g^{2}$, which is attractive for trionic correlations $(g>1)$. The propagator $D_{0}$ is given by

$$
\left(D_{0}^{-1}\right)_{i j}=\left(\begin{array}{cc}
G_{0} & F_{0} \\
F_{0}^{+} & -G_{0}^{+}
\end{array}\right)_{i j}^{-1},
$$

with $G_{0 i j}^{\alpha \beta} \equiv-\left\langle\hat{c}_{i \alpha} \hat{c}_{j \beta}^{+}\right\rangle_{B C S}$ and $F_{0 i j}^{\alpha \beta} \equiv-\left\langle\hat{c}_{i \alpha} \hat{c}_{j \beta}\right\rangle_{B C S}$.

Similar to [16], we can derive identities that relate all expectation values above to the full Green's function, $D_{i j} \equiv-\left\langle\Psi_{i} \bar{\Psi}_{j}\right\rangle_{\mathcal{S}}$ of the effective field theory. While we cannot compute $D_{i j}$ analytically in finite dimensions, a great simplification occurs in the $d \rightarrow \infty$ limit with $t \equiv t^{*} / \sqrt{d}$ (and $t^{*}=$ fixed), where the self energy becomes completely local [16]. In this limit, we can obtain $D_{i j}$ and thus compute $E\left(\Delta, g, n, n_{3}\right)$ exactly, by deriving self-consistent integral equations using an approach similar to dynamical mean field theory 16, 17].

The upper panel in Fig. 1 summarizes our results for the filling $\varrho \equiv n / 3=1 / 3$. We show the condensation 
energy per lattice site, defined as the energy difference between the superfluid state and the minimum energy state for the same filling and $\Delta=0$. For $|U|<\left|U_{C}\right| \approx$ $-1.774 t^{*}$, the minimum occurs at finite values of $\Delta$ and $g>1$; thus, we find a CSF with built-in three-body correlations. As expected, the energy of the CSF has a minimum with $n_{1}=n_{2}>n_{3}$. In other words, the 'spin operators' defined in terms of the the Gell-Mann matrices as $\hat{c}_{i}^{\dagger} \lambda^{\mu} \hat{c}_{i}$, tend to acquire a non-zero value, and the CSF becomes also "ferromagnetic". With an equal number of atoms of each color, this implies phase separation; that is, superfluid domains will form with the order parameter in each domain "pointing" in a different direction 18]. This tendency to form domains can also be captured through a Ginzburg-Landau analysis, to be discussed in a future publication [17]. Note that ferromagnetism appears only as a secondary order parameter, since the transition is driven by the local superfluid correlations.

The transition temperature $T_{C}$ associated with the formation of the CSF is related to the condensation energy, $E_{\text {cond }}$. As shown in Fig. 1. $E_{\text {cond }}$ initially increases with $|U|$; however, it goes to zero as one approaches the critical value, $U \rightarrow U_{C}$. At this point, the order parameter $\Delta$ also scales continuously to 0 ; thus, the point $U=U_{C}$ is a quantum critical point. At $U \rightarrow U_{C}$, the variational parameter $g$ diverges, and $|G\rangle$ reduces to a linear combination of states having trions (or no particles at all) at each lattice point. This $g \rightarrow \infty$ state provides the solution of minimum energy for the whole region $|U|>\left|U_{C}\right|$. Note also that in the high-dimensional limit, the effective hopping amplitude of the trions vanishes as $\sim 1 / d^{3 / 2}$. Therefore, trions are immobile in $d=\infty$ dimensions, and the energy of the trionic state is simply $E_{\text {trion }} / L=n U=3 \varrho U$, with $L$ the number of lattice sites. In $d=\infty$, the trionic state has also a finite residual entropy. This entropy is, however, immediately removed once one considers a finite dimensional system where trions can hop with some effective hopping $t_{\text {eff }} \neq 0$ : Then trions are expected to form a Fermi liquid with a Fermi temperature $T^{*}$. We expect the Fermi temperature to scale to 0 in finite dimensional lattices as one approaches $U_{C}$ from the trionic side. As shown in the inset of Fig. 1 the deviation from equal densities ("ferromagnetic polarization"), $\delta n_{3} \equiv \varrho-n_{3}$, also vanishes as $U \rightarrow U_{C}$, since the condensation energy that drives the accumulation of particles in the superfluid channels goes to zero at $U_{C}$.

This picture might, in principle, be modified through the intrusion of other phases. However, except for the half-filled fully-nested case not discussed here, there are no indications for this. At weak coupling, functional renormalization group [14] does not detect any other instabilities. The trionic repulsive Fermi liquid is apparently also stable, except possibly at $\rho \approx 1 / 2$, where charge ordering will take place. We therefore believe that our variational calculation captures the basic structure of the phase diagram, which, however, might become dec-
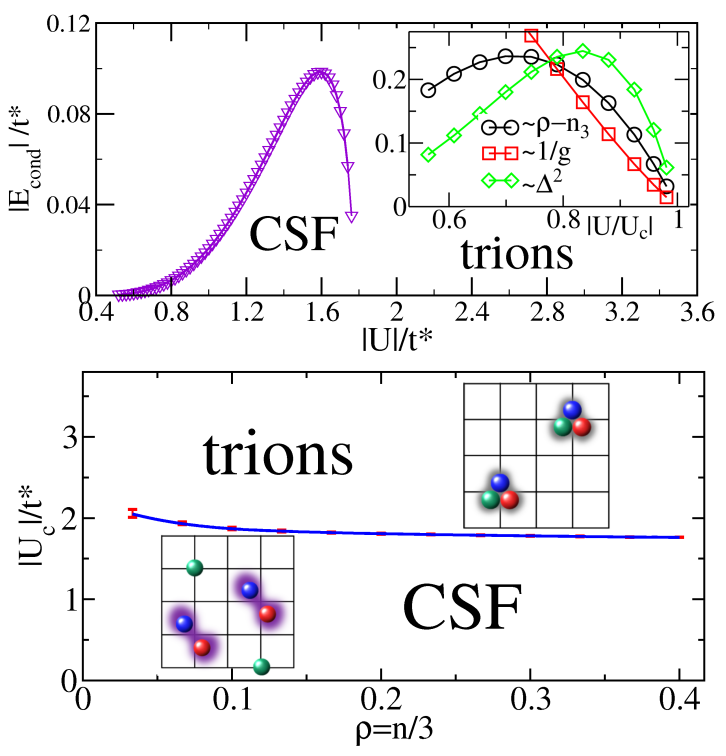

FIG. 1: (color online). Upper panel: Condensation energy as a function of $|U| / t^{*}$ for filling $\varrho \equiv n / 3=1 / 3$. Note that $t^{*}=t \sqrt{d}$ is the typical kinetic energy of the fermions and determines the Fermi temperature $T_{F}$. The inset shows how the superconducing order parameter, $\Delta$, the deviation from equal densities, $\delta n_{3} \equiv \varrho-n_{3}$, and $1 / g$ scale to zero as one approaches the critical value $U_{C} / t^{*} \approx-1.774$. Lower panel: $U_{C} / t^{*}$ as a function of the filling factor $\varrho=n / 3$. The phase diagram is symmetric to $\varrho=0.5$.

orated by subdominant and possibly undetectable instabilities at lower energy scales.

At $T>0$, thermal fluctuations destroy the CSF order above a critical temperature. In 3-dimensional lattices, $T_{C}$ is expected to be finite and related to the condensation energy $E_{\text {cond }}$. For $d<3$ dimensions, phase fluctuations completely destroy the long-range superconducting order at $T>0$.

The phase diagram in Fig. 1 parallels the famous finite temperature phase diagram of QCD where a superconducting state occurs at large quark densities, corresponding to large kinetic energies in our case, while baryonic matter emerges at low densities, i.e., small kinetic energies [19]. In QCD, however, the phase transition is believed to be of first order due to the long-range interaction generated by gluons. Furthermore, unlike quarks, the 3-color ultracold fermions considered here (e.g., ${ }^{6} \mathrm{Li}$ ) have no additional flavors and spin degrees of freedom. The CSF emerging in our case has a non-trivial SU(3) color content and is analogous to the color superconducting phase in 2-flavor QCD where only two flavors of light quarks are considered [20]. In the alternative theoretical scenario of 3-flavor QCD the superconducting state is expected to be color-flavor locked [21].

The variational analysis can be performed for all densities. We summarize our results in the $T=0$ phase diagram in the lower panel in Fig. 1. Because of particle-hole symmetry the phase diagram is symmetric with respect 
to half-filling, $\varrho=1 / 2$. Close to half filling, Fermi surface nesting could lead to additional phases not captured by our analysis.

Experimentally, the critical temperature could be reached by implementing adiabatic cooling in the optical lattice [8, 22]. To leading order, this procedure leaves the ratio $T / T_{F}$ invariant as absolute temperature scales decrease. From Fig. 1 we expect the CSF critical temperature to be $T_{C} / T_{F} \sim 0.1$ for the 3-color degenerate Fermi gas which has to be reached before the lattice is switched on. Realistic values for the dimensionless interaction strength are in the range $|U| / t \sim 1-100$. The full phase diagram can therefore be experimentally probed. Trion formation is expected to set in at temperatures of order $|U| \sim E_{R}$ where $E_{R} \approx 0.7 \mu K$ is the recoil energy of ${ }^{6} \mathrm{Li}$ in an optical lattice [10].

Maybe the easiest way to detect the CSF is to break the $\mathrm{SU}(3)$ symmetry to $\mathrm{U}(1)$ and detect vortices and the superconducting condensate fraction directly by pushing the system through a Feshbach resonance [11]. Furthermore, it should be also possible to observe the phase separation discussed above: For equal initial densities of all three internal states, domains will form where one of the states has a lower density than the others. This imbalance can be easily detected by light absorption, as already demonstrated for the BEC-BCS transition in a Fermi gas with overall spin imbalance [11]. Note that the system discussed here has equal total populations of all three hyperfine states, and the domain formation together with a local imbalance occurs spontaneously.

The quantum phase transition could also be observed via Bragg scattering [23]. With this technique, the dynamic structure factor $S(k, \omega)$ can be measured which is a suitable quantity for detecting the superfluid ground state [8]. In the CSF, several Goldstone modes arise due to the reduced $\mathrm{SU}(2)$ symmetry of the ground state, and one of them (the Anderson-Bogoliubov mode) is visible in $S(k, \omega)$ [14]. This mode will become soft close to the transition as the order parameter vanishes and disappears in the trionic phase.

The characteristic excitation spectrum of the trionic phase can be probed by "shaking" the optical lattice, i.e., applying a periodic amplitude modulation. This technique has already been used to determine the excitation spectrum of a bosonic Mott insulator [24, 25]. Deep in the trionic phase we expect a dominant excitation at a characteristic frequency $\omega=2|U|$ corresponding to the breaking up of trions, which becomes broadened by Fermi liquid quasiparticle excitations as one approaches the transition into the superfluid.

Since neither the CSF nor the trionic phase depends on a particular filling, we expect only small modifications of our results due to the trapping potential or the ensuing inhomogeneous density. Moreover, for $U \approx U_{C}$ the Cooper pair size $\xi$ will be small compared to the trap diameter $R$. Thus, the trapping potential plays a minor role as in the measurement of the dynamic structure factor for interacting bosons [23].

We thank D. Rischke, P. Zoller, and especially I. Bloch, E. Demler and M. Zwierlein for discussions and comments. We have been supported by Hungarian grants OTKA Nos. NF061726, T046267, and T046303, by the German Science Foundation (DFG) grant HO 2407/2-1, and the Alexander von Humboldt Foundation.

[1] See special issue on cold atoms, edited by K. Southwell, P. Campbell, and K. Ziemelis [Nature (London) 416, 206 (2002)].

[2] B. DeMarco and D. S. Jin , Science 285, 1703 (1999); A. G. Truscott et al., Science 291, 2570 (2001); F. Schreck et al., Phys. Rev. Lett. 87, 080403 (2001); Z. Hadzibabic et al., Phys. Rev. Lett. 88, 160401 (2002).

[3] S. Inouye et al., Nature 392, 151 (1998).

[4] D. Jaksch et al., Phys. Rev. Lett. 81, 3108 (1998)

[5] C. Orzel et al., Science 291, 2386 (2001).

[6] M. Greiner et al., Nature (London) 415, 39 (2002).

[7] M. Köhl et al., Phys. Rev. Lett. 94, 080403 (2005).

[8] W. Hofstetter et al., Phys. Rev. Lett. 89, 220407 (2002).

[9] C. A. Regal, M. Greiner, and D. S. Jin, Phys. Rev. Lett. 92, 040403 (2004); M. W. Zwierlein et al., Phys. Rev. Lett. 92, 120403 (2004); M. Bartenstein et al., Phys. Rev. Lett. 92, 120401 (2004).

[10] J. K. Chin et al., Nature (London) 443, 961 (2006).

[11] M. W. Zwierlein et al., Science 311, $492-496$ (2006); Y. Shin et al., Phys. Rev. Lett. 97, 030401 (2006).

[12] J. Stenger et al., Nature 396, 345 (1998); H. Schmaljohann et al., Phys. Rev. Lett. 92, 040402 (2004).

[13] C. A. Regal, PhD thesis, University of Colorado, Boulder (2005).

[14] C. Honerkamp and W. Hofstetter, Phys. Rev. Lett. 92, 170403 (2004); Phys. Rev. B 70, 094521 (2004).

[15] E.R.I. Abraham et al., Phys. Rev. A 55, R3299 (1997); M. Bartenstein et al., Phys. Rev. Lett. 94, 103201 (2005).

[16] W. Metzner and D. Vollhardt, Phys. Rev. Lett. 62, 324 (1989); Georges et al., Rev. Mod. Phys. 68, 13 (1996).

[17] A. Rapp et al., unpublished.

[18] Regarding phase separation, similar results have been obtained independently by R. Cherng, G. Refael, and E. Demler (unpublished).

[19] Z. Fodor and S. D. Katz, JHEP 0203, 014 (2002).

[20] M. Alford, K. Rajagopal and F. Wilczek, Phys. Lett. B 422, 247 (1998).

[21] M. Alford, K. Rajagopal and F. Wilczek, Nucl. Phys. B 537, 443 (1999).

[22] F. Werner et al., Phys. Rev. Lett. 95, 056401 (2005).

[23] D. M. Stamper-Kurn and W. Ketterle, in Coherent Atomic Matter Waves - Les Houches Summer School Session LXXII, edited by R. Kaiser, C. Westbrook, and F. David (Springer, 2001), p. 137; see also cond-mat/0005001.

[24] T. Stöferle et al., Phys. Rev. Lett. 92, 130403 (2004).

[25] C. Kollath et al., Phys. Rev. Lett. 97, 050402 (2006). 